\title{
La route difficile mais nécessaire vers la science ouverte
}

\begin{abstract}
La science ouverte est à la une, mais de quoi s'agit-il ? L'ambition est l'ouverture mondiale, immédiate et gratuite des publications scientifiques, ainsi que des données et codes associés. L'enjeu est d'avoir les résultats de la science accessibles à tous les niveaux de la société et sur tous les continents, aussi bien pour les chercheurs d'autres disciplines que pour les entreprises afin de booster l'innovation. Hélas, et malgré l'arrivée d'Internet, nous en sommes encore loin, que ce soit pour les données scientifiques et plus encore pour les articles. La majorité de nos revues s'appuient encore sur le modèle économique de la souscription. Sans abonnement, pas d'accès aux articles, et impossible de s'abonner à tout, c'est simplement trop cher, même pour un pays comme la France. La science ouverte est devenue une priorité politique, en Europe via le Plan S [1], en France via le plan national lancé par le ministère de l'Enseignement supérieur, de la Recherche et de l'Innovation il y a juste un an. La Société Française de Physique (SFP) soutient pleinement ces enjeux d'ouverture.
\end{abstract}

La communauté des physiciens et physiciennes a toujours été « ouverte » en ce qui concerne ses découvertes scientifiques, en déposant ses preprints dans les différentes archives ouvertes, telles que ArXiv et au niveau national HAL. À côté des archives, la SFP reconnait le rôle des maisons d'édition, ayant elle-même fondé les Éditions de Physique (devenues EDP Sciences) il y a près d'un siècle. Les maisons d'édition organisent la relecture par les pairs (peer review), un travail professionnel qui n'est pas fourni par les archives ouvertes. Le peer review constitue une étape dans l'édition scientifique, étape que vous êtes une grande majorité à estimer indispensable, comme vous pouvez le lire dans ce numéro qui présente (p. 50) l'enquête de la SFP sur ce sujet, menée en 2018 pendant les Journées de la Matière Condensée.

La science ouverte est un défı majeur, qui requiert une nouvelle façon de penser le partage de nos avancées scientifiques. Il y a des barrières psychologiques et économiques. L'ouverture des données est perçue comme lourde et inutile par de nombreux chercheurs. Or, c'est eux qui, les premiers, profitent de ces données bien documentées. L'édition scientifique est devenue un business complexe, qui s'est profondément mondialisé avec l'implication de quelques maisons d'édition puissantes dont les actionnaires attendent des retours sur investissement. II est donc plus que jamais nécessaire d'avoir une réflexion originale pour ouvrir la science, et une solidarité internationale qui rendent possible d'imposer cette ouverture aux grandes maisons d'édition.

Dans un communiqué de presse récent [2], la SFP s'est interrogée sur la lettre d'accord établie entre le consortium national Couperin et la multinationale Elsevier, couvrant presque un tiers de toutes les publications scientifiques en France. Cet accord s'appuie encore sur le modèle de souscription, et compte sur l'archive nationale HAL pour l'implémentation du libre accès. En Allemagne, universités et organismes de recherches, réunis dans le consortium DEAL, se mobilisent pour une nouvelle formule "publish and read". Certes, ce modèle n'est pas parfait non plus, mais il a le mérite d'esquisser un premier pas vers le libre accès pour tous. Pour l'instant, les négociations de DEAL avec Elsevier ont échoué.

La science ouverte constitue un enjeu important. La SFP est consciente qu'en France les différentes communautés ont des visions diverses sur l'évolution de l'édition scientifique. C'est une richesse et non un obstacle pour progresser. La croissance d'une «biblio-diversité » dans le paysage de l'édition scientifique est une condition sine qua non pour avancer ensemble, afin de créer demain un modèle durable adapté aux enjeux du $21^{\mathrm{e}}$ siècle.

Bart van Tiggelen $^{(a)}$ et Catherine Langlais ${ }^{(b)}$

(a) Président de la Commission des publications de la SFP

(b) Présidente de la Société Française de Physique

[1] B. van Tiggelen et al., Reflets de la physique 61 (2019) 48-51.

[2] www.sfpnet.fr/prise-de-position-de-la-sfp-sur-les-negociations-avec-elsevier 